\title{
SYDNEY UNIVERSITY NATURAL RADIOCARBON MEASUREMENTS IV
}

\author{
RICHARD GILLESPIE \\ Department of Physical Chemistry \\ University of Sydney, NSW 2006, Australia
}

The following list contains measurements made during the period 1972-5 which have not been previously published and which form part of a research project on marine shell dating (Gillespie, 1975). These sets of samples were measured to determine 1) "apparent age" of marine shells coll alive from Australian coastal waters before the advent of large-scale nuclear weapons testing, 2) the possibility of using post 1950 Australian marine shells as a modern reference, 3) "apparent age" of Australian marine shells in the past as shown by comparisons between stratigraphically equivalent charcoal and marine shell samples.

The procedures used were as described by Gillespie, Polach, and Temple (1972) and Gillespie and Temple (1973, 1976). Chemical pretreatment procedures are given in the text of each sec. In the absence of ${ }^{12} \mathrm{C} /{ }^{13} \mathrm{C}$ measurements, all charcoal samples were assumed to have $\delta^{13} \mathrm{C}=$ $-25 \pm 2 \%$ and all marine shell carbonate samples $\delta^{13} \mathrm{C}=0 \pm 2 \%$ relative to PDB (Craig, 1954; Polach, 1969; Lerman, 1972).

\section{ACKNOWLEDGMENTS}

The author would like to thank R B Temple for supervision and encouragement, H A Polach and his staff of the ANU Radiocarbon Laboratory for helpful advice and assistance throughout the project, J Chappell, Geography Department, ANU for statistical assistance, The Australian Museum, Macleay Museum, National Museum of Victoria, South Australian Museum, and Western Australian Museum for donations of shell samples, and $\mathrm{W}$ Ponder for identification of shell species. Part of this work was carried out while the author held a Commonwealth Post-Graduate Scholarship at the University of Sydney.

\section{SAMPLE DESCRIPTIONS}

\section{MARINE MOLLUSKS} Table 2.

Mollusk shells are represented throughout by code letters given in

\section{A. Marine mollusks collected alive before 1950}

Samples obtained from mus collns, pretreated by soaking for $48 \mathrm{hr}$ in $10 \%$ sodium hypochlorite solution to remove organic matter. Table 3 gives radiocarbon activities as $\delta^{14} \mathrm{C}$, the measured deviation from 0.95 NBS oxalic acid, age-corrected for period between colln and 1950 (Broecker and Olson, 1959).

The error-weighted mean of all measurements is $\delta^{14} \mathrm{C}=-8 \pm 4 \%$. When corrected for the Suess effect in mid-lat surface ocean waters by the method of Mangerud and Gulliksen (1975), the mean value is $\delta^{14} \mathrm{C}=$ 
TABLE 1

Interlaboratory crosschecks

\begin{tabular}{lclcc}
\hline Lab no. & SUA date & Other no. & Other date & Ref \\
\hline SUA-191/2 & $610 \pm 80$ & ANU-1625 & $640 \pm 70$ & $\begin{array}{l}\text { Polach } \\
\text { (pers commun) }\end{array}$ \\
SUA-233/2 & $300 \pm 80$ & ANU-1626 & $340 \pm 70$ &,$"$ \\
SUA-263/2 & $450 \pm 80$ & ANU-1627 & $600 \pm 70$ &,$"$ \\
SUA-380 & $1870 \pm 100$ & NSW-96 & $1990 \pm 100$ & $\begin{array}{l}\text { Carswell } \\
\text { (pers commun) }\end{array}$ \\
& & & &
\end{tabular}

TABLE 2

Species names for mollusks

\begin{tabular}{llll}
\hline Code & \multicolumn{1}{c}{ Species } & Code & \multicolumn{1}{c}{ Species } \\
\hline A & Anadara trapezia & N & Notohaliolis ruber \\
B & Austrocochlea constricta & O & Nerita atramentosa \\
G & Austrocochlea rudis & P & Patellanax peroni \\
D & Bankivia fasciata & Q & Pinctada margaritifera \\
E & Bembicium melanostomum & R & Pinna bicolor \\
F & Brachidontes rostratus & S & Pitar sp \\
G & Cabestana spengleri & T & Plebinonax deltoides \\
H & Cellana solida & $\mathrm{U}$ & Proxichione laqueata \\
I & Conuber incei & V & Pyrazus ebinezus \\
J & Katelysia rhytiphora & W & Saccostrea cucculata \\
K & Littorina unifasciata & X & Thais orbita \\
L & Mactra obesa & Y & Turbo (Ninella) torquata \\
M & Mytilus edulis planulatus & Z & Turbo (Subninella) undulata. \\
\hline
\end{tabular}

TABLE 3

Marine mollusk collected alive before 1950

\begin{tabular}{lllcc}
\hline Lab no. & \multicolumn{1}{c}{ Location } & Colln yr & $\begin{array}{c}\text { Species } \\
(\text { Table })\end{array}$ & $\delta^{14} \mathrm{C} \%$ \% \\
\hline SUA-354/1 & $\begin{array}{l}\text { Torres Strait } \\
\left(\text { ca } 10^{\circ} \mathrm{S}, 143^{\circ} \mathrm{E}\right)\end{array}$ & $1875 \pm 3$ & $\mathrm{~L}$ & $-8 \pm 8$ \\
SUA-354/2 & $\begin{array}{l}\text { Torres Strait } \\
\left(\text { ca } 10^{\circ} \mathrm{C}, 143^{\circ} \mathrm{E}\right)\end{array}$ & $1875 \pm 3$ & $\mathrm{R}$ & $-6 \pm 10$ \\
SUA-357 & $\begin{array}{l}\text { Torres Strait } \\
\left(\text { ca } 10^{\circ} \mathrm{S}, 143^{\circ} \mathrm{E}\right)\end{array}$ & 1909 & $\mathrm{Q}$ & $+1 \pm 10$ \\
SUA-355 & $\begin{array}{l}\text { Garden Is, WA } \\
\left(32^{\circ} 15^{\prime} 5,115^{\circ} 40^{\prime} \mathrm{E}\right)\end{array}$ & 1930 & $\mathrm{U}$ & $-5 \pm 10$ \\
SUA-393 & $\begin{array}{l}\text { Adelaide, SA } \\
\left(\text { ca } 35^{\circ} \mathrm{S}, 13^{\circ} \mathrm{E}\right)\end{array}$ & $1937 \pm 2$ & $\mathrm{~T}$ & $-20 \pm 10$ \\
SUA-356 & $\begin{array}{l}\text { Narooma, NSW } \\
\left(36^{\circ} 13^{\prime} \mathrm{S}, 150^{\circ} 07^{\prime} \mathrm{S}\right)\end{array}$ & 1950 & $\mathrm{~J}$ & $-8 \pm 10$ \\
\hline
\end{tabular}


$-5 \pm 4 \%$, equivalent to $\Delta=-55 \pm 4 \%$ and an "apparent age" of $450 \pm 35$ yr for Australian coastal waters.

\section{B. Marine mollusks collected alive in 1973}

Table 4 lists the radiocarbon activity in percentage modern for marine mollusk shells coll alive from Australian coastal waters in 1973. These measurements fall into 2 groups, each having a normal distribution; samples from $\mathrm{E}$ coast with a mean activity $106.8 \pm 2.3 \%$ modern, and samples from $\mathrm{S}$ coasts with mean activity $117.4 \pm 4.5 \%$ modern. Difference between means is significant at $0.1 \%$ level by t test. Activities from Australian E coast compare with those from Makara, New Zealand (Rafter, pers commun) of $110.7 \pm 0.7 \%$ modern. Different activities on $\mathrm{S}$ and $\mathrm{E}$ coasts may be explained by different surface ocean currents supplying these regions (Knox, 1963).

The range of activities between species at same location, and for same species at different locations are consistent with results from Baltic Sea (Erlenkeuser \& Willkomm, 1973; Erlenkeuser et al, 1975) and indicate that these mollusk shells cannot be used to estimate a modern ref for marine carbonate.

\section{ARCHAEOLOGIC SAMPLES}

Measurements on charcoal and marine shells from Australian coastal middens, made to determine relationship between radiocarbon ages of stratigraphically equivalent terrestrial organic and marine shell carbon. Shell species names in Table 2. Charcoal pretreated with dilute phosphoric acid and sodium hydroxide/sodium pyrophosphate solutions (Goh and Molloy, 1972). Shells etched in dilute hydrochloric acid to remove ca $15 \%$ by weight of surface carbonate.

\section{Bass Point series}

Midden on Bass Point, N S W (34 $36^{\prime}$ S, $150^{\circ} 46^{\prime}$ E) excavated 1972 by $\mathrm{R}$ Gillespie and $\mathrm{P}$ J Hughes, Dept Prehist, Australian Natl Univ, who reported on depositional environment (Lampert and Hughes, 1974). Previous excavation at site reported by Bowdler (1970).

SUA-47.

Charcoal 10 to $15 \mathrm{~cm}$ below surface.

SUA-45/S1.

Shell Species M, 10 to $15 \mathrm{~cm}$.

SUA-45/S2.

Shell Species P, 10 to $15 \mathrm{~cm}$.

SUA-45/S3

Shell Species Y, 10 to $15 \mathrm{~cm}$.
$320 \pm 75$

AD 1630

$725 \pm 65$

AD 1225

$680 \pm 65$

AD 1270

$695 \pm 65$

AD 1255 
SUA-45/S4.

Shell Species G, 10 to $15 \mathrm{~cm}$.

SUA-146.

Charcoal, 30 to $40 \mathrm{~cm}$.

SUA-145/S1.

Shell Species M, 30 to $35 \mathrm{~cm}$.

SUA-145/S2.

Shell Species P, 30 to $35 \mathrm{~cm}$.

SUA-145/S3A.

Shell Species $Y>3 \mathrm{~cm}$ diam, 30 to $35 \mathrm{~cm}$.

SUA-145/S3B.

Same, $<3 \mathrm{~cm}$ diam, 30 to $35 \mathrm{~cm}$.

SUA-145/S3C.

Operculae of same, 30 to $35 \mathrm{~cm}$.

SUA-145/S4.

Shell Species N, 30 to $35 \mathrm{~cm}$.

SUA-145/S5.

Shell Species G, 30 to $35 \mathrm{~cm}$.

SUA-145/S6.

Shell Species X, 30 to $35 \mathrm{~cm}$.

SUA-25.

Charcoal, 50 to $60 \mathrm{~cm}$.

SUA-24/S1A.

Shell Species Y, 50 to $55 \mathrm{~cm}$, no pretreatment.

SUA-24/S1B.

Same, with standard acid etching pretreatment.

SUA-24/S2A.

Shell Species G, 50 to $55 \mathrm{~cm}$, no pretreatment.

SUA-24/S2A.

Same, standard pretreatment.
$665 \pm 70$

AD 1285

$985 \pm 100$ AD 965

$$
\begin{array}{r}
1100 \pm 65 \\
\text { AD } 850 \\
1250 \pm 65 \\
\text { AD } 700
\end{array}
$$

$$
\begin{array}{r}
1290 \pm 65 \\
A D 660 \\
1290 \pm 65 \\
A D 660 \\
1285 \pm 65 \\
\text { AD } 665
\end{array}
$$

$$
\begin{array}{r}
1270 \pm 75 \\
\text { AD } 680
\end{array}
$$

$$
1130 \pm 75
$$$$
\text { AD } 820
$$

$$
1190 \pm 75
$$
AD 760

$2650 \pm 70$ $700 \mathrm{BC}$

$$
2480 \pm 70
$$

530 BC

$2870 \pm 80$

920 BC

$1990 \pm 80$ $40 \mathrm{BC}$

$2180 \pm 80$ $230 \mathrm{BC}$ 
TABLE 4

Marine mollusks collected alive in 1973

\begin{tabular}{|c|c|c|c|}
\hline Lab no. & Location & $\begin{array}{c}\text { Species } \\
\text { (see Table 2) }\end{array}$ & $\begin{array}{l}{ }^{14} \mathrm{C} \text { activity } \\
\% \text { modern } \pm 1 \sigma\end{array}$ \\
\hline SUA-23/1 & $\begin{array}{l}\text { Bass Point NSW } \\
\left(34^{\circ} 36^{\prime} \mathrm{S}, 150^{\circ} 46^{\prime} \mathrm{E}\right)\end{array}$ & M & $103.7 \pm 0.6$ \\
\hline SUA-23/2 & " $\quad "$ & $\mathbf{P}$ & $107.7 \pm 0.6$ \\
\hline SUA-23/3 & $"$ & $\mathrm{Y}$ & $107.4 \pm 0.6$ \\
\hline SUA-23/4 & " & $\mathrm{G}$ & $105.2 \pm 0.9$ \\
\hline SUA-23/5 & , & $\mathrm{W}$ & $108.7 \pm 0.6$ \\
\hline SUA-23/6 & ", & $\mathrm{O}$ & $109.6 \pm 1.1$ \\
\hline SUA-27/1 & $\begin{array}{l}\text { Cottlesloe, WA } \\
\left(32^{\circ} 01^{\prime} \mathrm{S}, 115^{\circ} 45^{\prime} \mathrm{E}\right)\end{array}$ & $\mathrm{C}$ & $116.9 \pm 0.9$ \\
\hline SUA-27/2 & " & $\mathrm{K}$ & $113.4 \pm 1.2$ \\
\hline SUA-27/3 & $\begin{array}{l}\text { Fremantle, WA } \\
\left(32^{\circ} 03^{\prime} \mathrm{S}, 115^{\circ} 47^{\prime} \mathrm{E}\right)\end{array}$ & $\mathrm{E}$ & $124.0 \pm 1.3$ \\
\hline SUA-209/1 & $\begin{array}{l}\text { Moruya, NSW } \\
\left(35^{\circ} 54^{\prime} \mathrm{S}, 150^{\circ} 07^{\prime} \mathrm{E}\right)\end{array}$ & $\mathrm{T}$ & $107.1 \pm 0.9$ \\
\hline SUA-209/2 & ", & $\mathrm{D}$ & $104.1 \pm 0.9$ \\
\hline SUA-218/1 & $\begin{array}{l}\text { Macleay Is Qld } \\
\left(27^{\circ} 39^{\prime} \mathrm{S}, 153^{\circ} 22^{\prime} \mathrm{E}\right)\end{array}$ & $\mathbf{M}$ & $105.9 \pm 0.8$ \\
\hline SUA-218/2 & ", & V & $104.6 \pm 0.8$ \\
\hline SUA-220 & $\begin{array}{l}\text { Broadwater, NSW } \\
\left(29^{\circ} 00^{\prime} \mathrm{S}, 153^{\circ} 29^{\prime} \mathrm{E}\right)\end{array}$ & I & $109.9 \pm 0.9$ \\
\hline SUA-273/1 & $\begin{array}{l}\text { Swan Bay, Victoria } \\
\left(38^{\circ} 17^{\prime} \mathrm{S}, 144^{\circ} 40^{\prime} \mathrm{E}\right)\end{array}$ & $\mathrm{J}$ & $118.6 \pm 1.0$ \\
\hline SUA-273/2 & ", & $\mathrm{Z}$ & $118.6 \pm 0.9$ \\
\hline SUA-294/1 & $\begin{array}{l}\text { Bruny Is Tas } \\
\left(43^{\circ} 21^{\prime} \mathrm{S}, 147^{\circ} 20^{\prime} \mathrm{E}\right)\end{array}$ & $\mathrm{M}$ & $110.9 \pm 0.9$ \\
\hline SUA-294/2 & " " & $\mathrm{H}$ & $114.6 \pm 0.9$ \\
\hline SUA-294/3 & $", \quad "$ & $\mathrm{~B}$ & $111.3 \pm 1.1$ \\
\hline SUA-300/1 & $\begin{array}{l}\text { Noarlunga, SA } \\
\left(35^{\circ} 10^{\prime} \mathrm{S}, 138^{\circ} 28^{\prime} \mathrm{E}\right)\end{array}$ & $\mathrm{H}$ & $121.9 \pm 1.0$ \\
\hline SUA-300/2 & ", & $\mathrm{N}$ & $122.2 \pm 1.0$ \\
\hline SUA-311 & $\begin{array}{l}\text { Pt Stuart, NT } \\
\left(12^{\circ} 13^{\prime} \mathrm{S}, 131^{\circ} 53^{\prime} \mathrm{E}\right)\end{array}$ & W & $117.6 \pm 1.0$ \\
\hline
\end{tabular}

General Comment: shell ages from 10 to $15 \mathrm{~cm}$ level in good agreement, mean age difference from charcoal, $+379 \mathrm{yr}$. Similarly, mean difference for 30 to $35 \mathrm{~cm}$ level, +241 yr. For 50 to $55 \mathrm{~cm}$ level, shell ages SUA-24/ $\mathrm{S} 1 \mathrm{~A}$ and $\mathrm{B}$ indicate standard pretreatment necessary and effective for re- 
moving younger contamination, SUA-24/S2A and B, partly recrystallized (22\% calcite), pretreatment not effective.

\section{Jervis Bay series}

Midden at Cemetery Point (35 $\left.10^{\prime} \mathrm{S}, 150^{\circ} 32^{\prime} \mathrm{E}\right)$ excavated 1973 by R J Lampert, Prehist Dept, Australian Natl Univ.

\section{SUA-260C. CPIB/10}

Charcoal, $65 \mathrm{~cm}$ below surface.

SUA-260/S1.

Shell Species M, $65 \mathrm{~cm}$.

SUA-260/S2.

Shell Species P, $65 \mathrm{~cm}$.

SUA-261C. CP1B/15

Charcoal, $104 \mathrm{~cm}$.

SUA-261/SI.

Shell Species Y, $104 \mathrm{~cm}$.

SUA-261/S2.

Shell Species M, $104 \mathrm{~cm}$.

SUA-262C. CP1B/22

Charcoal, $148 \mathrm{~cm}$.

SUA-262/S1.

Shell Species Y, $148 \mathrm{~cm}$.

SUA-262/S2.

Shell Species O, $148 \mathrm{~cm}$.

SUA-262/S3.

Shell Species P, $148 \mathrm{~cm}$.

\section{SUA-262/S4.}

Shell Species M, $148 \mathrm{~cm}$.

$$
\begin{array}{r}
390 \\
\text { AD } 1560
\end{array}
$$

$$
\begin{array}{r}
800 \\
\text { AD } 1150
\end{array}
$$

$$
720 \pm 60
$$

AD 1230

$$
\begin{array}{r}
1790 \pm 90 \\
\text { AD } 160
\end{array}
$$

$$
\begin{array}{r}
970 \pm 75 \\
\text { AD } 980 \\
970 \pm 75 \\
\text { AD } 980
\end{array}
$$

$$
\begin{array}{r}
910 \pm 60 \\
\text { AD } 1040 \\
1285 \pm 70 \\
\text { AD } 665
\end{array}
$$

$$
1275 \pm 80
$$

AD 675

$$
1125 \pm 80
$$

AD 825

$$
1265 \pm 70
$$

AD 685

General Comment (RJL): only SUA-261C is anomalous within series. Aboriginal occupants of site probably burnt stranded driftwood on their fires, some of which were perhaps old at time of colln. Shell dates, then, forming very consistent series and agreeing well with other charcoal dates, may be more reliable indicators of antiquity at coastal midden sites. Mean age difference for $65 \mathrm{~cm}$ level is $+370 \mathrm{yr}$, and for $148 \mathrm{~cm}$ level is $+328 \mathrm{yr}$. 


\section{Tamboon Inlet series}

Midden in E Victoria ( $37^{\circ} 47 \mathrm{~S}, 149^{\circ} 17^{\prime} \mathrm{E}$ ) excavated 1974 by $\mathrm{P} \mathrm{J} \mathrm{F}$ Coutts, Archaeol \& Aboriginal Relics Office, Melbourne.

$360 \pm 85$

SUA-377C.

Charcoal, 40 to $50 \mathrm{~cm}$ below surface.

AD 1590

SUA-377S.

Shell Species F, 40 to $50 \mathrm{~cm}$.

SUA-378C.

Charcoal, 10 to $20 \mathrm{~cm}$.

SUA-378S.

Shell Species F, 10 to $20 \mathrm{~cm}$.
$660 \pm 95$ AD 1290

$$
220 \pm 85
$$

AD 1730

AD 1530

$420 \pm 100$
Old Beach series

Midden on E side of Derwent Estuary, Tasmania $\left(42^{\circ} 46^{\prime} 25^{\prime \prime} \mathrm{S}\right.$, $\left.147^{\circ} 15^{\prime} 30^{\prime \prime} \mathrm{E}\right)$ excavated 1973 by W R Sigleo, Geog Dept, Univ Tasmania.

SUA-306. OB001

$5800 \pm 130$

3850 BC

Charcoal, $35 \mathrm{~cm}$ below surface.

SUA-307. 0B002

$6010 \pm 90$

$4060 \mathrm{BC}$

Shell Species F, $35 \mathrm{~cm}$.

\section{Swansea Inlet series}

Midden on Swansea Channel, near Newcastle, N S W $\left(33^{\circ} 06^{\prime} \mathrm{S}\right.$, $\left.151^{\circ} 40^{\prime} \mathrm{E}\right)$ excavated by L K Dyall, Chem Dept, Univ Newcastle.

SUA-238C.

$1965 \pm 90$

Charcoal, 0 to $4 \mathrm{~cm}$ below surface.

15 BC

SUA-238/S1.

$2690 \pm 90$

$740 \mathrm{BC}$

Shell Species W, 0 to $4 \mathrm{~cm}$.

SUA-238/S2.

$2480 \pm 90$

Shell Species A, 0 to $4 \mathrm{~cm}$.

General Comment: shell ages SUA-238/S1 and 2 agree well, mean age difference from charcoal SUA-238C is $+620 \mathrm{yr}$.

\section{Broughton Island series}

Midden on small island off N S W coast $\left(32^{\circ} 35^{\prime} \mathrm{S}, 152^{\circ} 20^{\prime} \mathrm{E}\right)$ excavated 1974 by R V S Wright, Anthropol Dept, Sydney Univ. 


\section{SUA-402C.}

Charcoal, 50 to $60 \mathrm{~cm}$ below surface.

SUA-402/S1.

Shell Species O, 50 to $60 \mathrm{~cm}$.

SUA-402/S2.

Shell Species P, 50 to $60 \mathrm{~cm}$.
$445 \pm 179$

AD 1505

$$
420 \pm 85
$$

AD 1530

$$
600 \pm 85
$$

General Comment: shell ages SUA-402/S1 anad 2 agree well, mean age difference from charcoal SUA-402C is $+65 \mathrm{yr}$.

\section{Connection Creek I series}

Midden in lower Macleay Valley, N S W $\left(31^{\circ} 15^{\prime} \mathrm{S}, 152^{\circ} 55^{\prime} \mathrm{E}\right)$ excavated 1972 by G E Connah, Prehist Dept, Univ New England.

SUA-395C. CCI.73.62

$3720 \pm 100$

Charcoal, 50 to $60 \mathrm{~cm}$ below surface.

SUA-395S. CCI.73.65

$3750 \pm 100$

Shell Species W, 50 to $60 \mathrm{~cm}$.

SUA-396C. CCI.73.132

$3400 \pm 100$

Charcoal, 100 to $110 \mathrm{~cm}$.

SUA-396S. CCI.73.141

$3790 \pm 100$

Shell Species A, 100 to $110 \mathrm{~cm}$.

\section{$1840 \mathrm{BC}$}

\section{Hooka Point series}

Further samples from midden near Lake Illawarra, N S W $\left(34^{\circ} 30^{\prime}\right.$ S, $\left.150^{\circ} 51^{\prime} \mathrm{E}\right)$ excavated 1973 by $\mathrm{J}$ P White, Anthropol Dept, Sydney Univ. Other samples from site pub in R, 1976, v 18, p 96-109.

\section{SUA-65/1. DK13/7}

Shell Species A, $10 \mathrm{~cm}$ below surface.

SUA-65/2. DL/DM 13/9C

Shell Species A, $40 \mathrm{~cm}$.

\section{SUA-65/3.}

Shell Species A, $80 \mathrm{~cm}$.

$$
5650 \pm 85
$$

3700 BC

$$
\begin{aligned}
& 5230 \pm 85 \\
& 3280 \text { BC }
\end{aligned}
$$

$$
5570 \pm 85
$$

3620 BC

General Comment: ages not related to depth due to reworking of midden material by storm waves (Emmerson, 1973; Hughes and Sullivan, 1974). 


\section{Currarong II series}

Rock shelter midden near Currarong, N S W (35 $\left.01^{\prime} \mathrm{S}, 150^{\circ} 49^{\prime} \mathrm{E}\right)$ excavated 1973 by P J Hughes.

SUA-241/1.

$2040 \pm 70$

90 BC

Shell species W, 30 to $40 \mathrm{~cm}$ below surface.

SUA-241/2.

$1600 \pm 70$

Shell Species V, 30 to $40 \mathrm{~cm}$.

SUA-241/3.

$1660 \pm 70$

Shell Species Y, 30 to $40 \mathrm{~cm}$.

SUA-242/1.

Shell Species W, 80 to $90 \mathrm{~cm}$.

SUA-242/2.

$3670 \pm 90$

Shell Species V, 80 to $90 \mathrm{~cm}$.

SUA-224.

$5990 \pm 80$

4040 BC

Shell Species W, 100 to $120 \mathrm{~cm}$.

General Comment: good agreement between shell ages from some levels, may be compared with charcoal ages from different excavation at this site: NSW-76, $37.5 \mathrm{~cm}, 1520 \pm 100$ (Djohadze, pers commun), ANU-386, $105 \mathrm{~cm} 3740 \pm 100$ (Lampert, 1971).

All dates in this sec indicate that aboriginal shell middens are not ideal sites for determination of past values for "apparent age" of marine shell carbonate by comparison with stratigraphically equivalent charcoal. The range of age differences between shell and charcoal samples is consistent with remarks of Ambrose (1967) and Hughes and Sullivan (1974) about potential hazards of midden archaeol. Other sites where deposition of terrestrial organic and marine carbonate samples can be considered contemporaneous are needed.

\section{REFERENCES}

Ambrose, W R, 1967, Archaeology and shell middens: Archaeol \& Phys Anthropol in Oceania, v 2, p 169-187.

Bowdler, S, 1970, Bass Point: the excavation of a South-East Australian shell midden showing cultural and economic change: Unpub BA thesis, Univ Sydney.

Broecker, W S and Olson, E A, 1959, Lamont radiocarbon measurements VI: Radiocarbon, v 1, p 111-132.

1961, Lamont radiocarbon measurements VIII: Radiocarbon, v 3, p 176-204.

Craig, Harmon, 1954, Carbon-13 in plants and the relationship between carbon-13 and carbon-14 in Nature: Jour Geol, v 62, p 115-148.

Emmerson, P, 1973, Hooka Point: a disturbed site: Unpub BA thesis, Univ Sydney.

Erlenkeuser, H and Willkomm, H, 1973, University of Kiel radiocarbon measurements VII: Radiocarbon, v 15, p 113-126.

Erlenkeuser, H, Metzner, H, and Willkomm, H, 1975, University Kiel radiocarbon measurements VIII: Radiocarbon, v 17, p 276-300. 
Gillespie, Richard, 1975, The suitability of marine shells for radiocarbon dating: Unpub PhD thesis, Univ Sydney.

Gillespie, R, Polach, H A, and Temple, R B, 1972, Sydney University natural radiocarbon measurements I: Radiocarbon, v 14, p. 413-417.

Gillespie, R and Temple, R B, 1973, Sydney University natural radiocarbon measurements II: Radiocarbon, v 15, p 566-573.

1976, Sydney University natural radiocarbon measurements III: Radiocarbon, v 18, p 96-109.

Goh, K M and Molloy, B P J, 1972, Reliability of radiocarbon dates from buried charcoals: 8th internatl radiocarbon conf Proc, New Zealand, p G29-45.

Hughes, P J and Sullivan, M E, 1974, The redeposition of midden material by storm waves: Jour Royal Soc NSW, v 107, p 6-10.

Knox, G A, 1963, The biogeography and intertidal ecology of the Australian coasts: Annual Rev Oceanog Marine Biol, v 1, p 341-404.

Lampert, R J, 1971, Burrill Lake and Currarong: coastal sites in southern NSW: Terra Australis, v 2, ANU Prehist Dept.

Lampert, R J and Hughes, P. J, 1974, Sea level change and aboriginal coastal adaptations in southern NSW: Archaeol \& Phys Anthropol in Oceania, v 9, p 226-235.

Lerman, J C, 1972, Carbon dating: origin and correction of isotope fractionation errors in terrestrial living matter: 8th internatl radiocarbon conf Proc, New Zealand, p H16-28.

Mangerud, J and Gulliksen, S, 1975, Apparent radiocarbon ages of recent marine shells from Spizsbergen, Norway and Arctic Canada: Quaternary Research, v 5, p 263-273.

Polach, H A, 1969, Optimization of liquid scintillation radiocarbon age determinations and reporting of ages: Atomic energy in Australia, v 12, p. 21-28. 